Cite this: Phys. Chem. Chem. Phys., 2014, 16, 2280

Received 19th November 2013, Accepted 14th December 2013

DOI: $10.1039 / c 3 c p 54877 e$

www.rsc.org/pccp

\section{Nitrogen-doped reduced graphene oxide electrodes for electrochemical supercapacitors $\dagger$}

\author{
Hugo Nolan, ${ }^{\text {ab }}$ Beatriz Mendoza-Sanchez, ${ }^{a}$ Nanjundan Ashok Kumar, ${ }^{a}$ \\ Niall McEvoy, ${ }^{a}$ Sean O'Brien, ${ }^{\text {ac }}$ Valeria Nicolosi ${ }^{\mathrm{abc}}$ and Georg S. Duesberg ${ }^{\star a b}$
}

\begin{abstract}
Herein we use Nitrogen-doped reduced Graphene Oxide (N-rGO) as the active material in supercapacitor electrodes. Building on a previous work detailing the synthesis of this material, electrodes were fabricated via spray-deposition of aqueous dispersions and the electrochemical charge storage mechanism was investigated. Results indicate that the functionalised graphene displays improved performance compared to non-functionalised graphene. The simplicity of fabrication suggests ease of up-scaling of such electrodes for commercial applications.
\end{abstract}

Recent research has shown that the unique physical, chemical and electronic properties of graphene may be exploited in a wide range of applications; namely sensing, ${ }^{1,2}$ electronics ${ }^{3,4}$ and energy. ${ }^{5,6}$ In particular, energy conversion and storage technologies that take advantage of graphene's excellent mechanical strength, chemical stability and high surface area may be developed. Indeed, many research publications detail the use of graphene and related materials in lithium ion battery electrodes, ${ }^{7}$ solar energy conversion, ${ }^{8}$ supercapacitors ${ }^{9}$ and as electrode materials in other electrochemical energy devices. ${ }^{10}$ For many of these applications it has been shown that the presence of heteroatoms in the graphene lattice improves material performance. Chemical modification allows for tuning of graphene properties such as surface chemistry and electronic properties; ${ }^{11}$ which renders them more suited to certain applications than pristine graphene. Nitrogen-doped (N-doped) graphene has been demonstrated to be of use as an electrocatalytic material for oxygen reduction in hydrogen fuel cells, ${ }^{12}$ improves biocompatibility of carbon devices in biosensing ${ }^{13}$ and enhances the performance of graphene-based supercapacitors. ${ }^{14}$

\footnotetext{
${ }^{a}$ Centre for Research on Adaptive Nanostructures and Nanodevices (CRANN) \& Advanced Materials Bio-Engineering, Research Centre (AMBER), Trinity College Dublin, Dublin 2, Ireland. E-mail: duesberg@tcd.ie; Fax: +35318963037

${ }^{b}$ School of Chemistry, Trinity College Dublin, Dublin 2, Ireland

${ }^{c}$ School of Physics, Trinity College Dublin, Dublin 2, Ireland

$\dagger$ Electronic supplementary information (ESI) available: Electrochemical comparison of N-rGO electrodes with untreated GO electrodes, X-ray Photoelectron Spectroscopy in detail, further electrochemical measurements of N-rGO, BET analysis, XRD comparison of GO and N-rGO. See DOI: 10.1039/c3cp54877e
}

Graphene may be synthesised via mechanical cleavage of graphite flakes, ${ }^{15}$ Chemical Vapour Deposition (CVD) ${ }^{16}$ or the decomposition of silicon carbide (SiC). ${ }^{17}$ However, while the latter two have great potential in electronics fabrication, it is not feasible to produce gram-scale quantities using these methods. Liquid phase exfoliation and processing of graphene is one method which shows great potential as a means of producing large quantities of material. ${ }^{18,19}$ Reduction of graphene oxide (GO) is one method which shows promise as a means of producing large quantities of material suited for energy applications. ${ }^{20-22}$ Graphite oxide is easily exfoliated and the oxygen functional groups can be removed via thermal or chemical means. While the reduction of GO produces a graphene material with structural defects and residual heteroatoms, these issues may not be problematic for applications such as catalysis and energy storage, where crystallinity and structural integrity of the graphene material is not a priority. In fact, the presence of a few oxygen-containing groups at the surface can advantageously influence the interfacial activity between graphene electrodes and the electrolyte.

Much work has been carried out towards production of $\mathrm{N}$-doped graphene via several methods. Reported techniques include in situ processes and post-growth treatments to obtain $\mathrm{N}$-doped CVD graphene, ${ }^{23-26}$ while other studies show that GO-sourced N-doped graphene may be obtained through various methods. ${ }^{27-29}$ High temperature treatments, required for thermal reduction of GO, can lead to the removal of desired nitrogen functional groups. Plasma treatments have been successfully demonstrated as an alternative route towards the functionalisation of reduced $\mathrm{GO}(\mathrm{rGO})$. Often such processes require several steps to achieve both reduction and functionalisation. Studies by both Jafri et al. ${ }^{30}$ and Jeong et al. ${ }^{14}$ employ a combination of nitrogen plasma and mild thermal treatment to produce N-doped rGO (N-rGO).

Recent work has shown that ammonia plasma treatments may be employed to simultaneously reduce and N-dope GO at room temperature. ${ }^{31}$ This technique boasts a one-step process which negates the requirement for corrosive or toxic chemicals or high temperature treatments during the reduction process. As it has widely been reported in the literature that $\mathrm{N}$-doped 
graphene enhances the performance of graphene-based supercapacitors, we propose that the N-rGO produced via ammonia plasma treatment of GO is suitable for such an application. Herein we demonstrate the application of $\mathrm{N}-\mathrm{rGO}$ as the active material in supercapacitor electrodes produced via spraydeposition onto conductive substrates and investigate the charge storage mechanism. We propose that our production process boasts advantages over existing techniques by virtue of the fact that no binder or conductivity enricher are required in the active material, use of hazardous solvents is eliminated by using aqueous dispersions and no chemical or high temperature processing is required in the reduction and doping of the N-rGO.

Sample preparation has previously been reported ${ }^{31}$ but shall be summarised herein. In brief, GO powder was synthesised by a modified Hummers and Offeman method ${ }^{32}$ to produce the starting material. Gram scale quantities of this material were placed in a chamber downstream from a radical plasma generator at $1000 \mathrm{~W}$ with a gas flow of $100 \mathrm{sccm}$ of a $1: 1$ mixture of $\mathrm{H}_{2}$ and $\mathrm{NH}_{3}$. No direct heating of the GO took place and the lack of applied bias in the vicinity of the sample minimised kinetic damage from accelerated ions. The energetically relaxed but chemically active plasma allows reactions between ammonia radicals and oxygen moieties on the GO, removing these and substitutionally functionalising the material with nitrogen.

$\mathrm{N}-\mathrm{rGO}$ electrodes were fabricated by spray-depositing aqueous dispersions of the material onto substrates. This was accomplished by dispersing the N-rGO in water and tip sonicating for one hour to create a stable aqueous dispersion which was then spray-coated onto conductive ITO/glass substrates using a Prism Ultracoat 300 commercial spray tool to a thickness of $\sim 700 \mathrm{~nm}$. Typical mass loadings were $0.05-0.07 \mathrm{mg}$ per electrode. Substrates were heated to $100{ }^{\circ} \mathrm{C}$ during spray deposition and a thin adhesion layer of polyethylenimine (PEI) was initially sprayed on all substrates as previously reported ${ }^{33}$ to improve the mechanical robustness of the deposited film. A Carl Zeiss FE-SEM Ultra was employed using an in-lens electron detector. Raman spectroscopy was carried out with a Witec Alpha $300 \mathrm{R}$ confocal Raman microscope and X-ray Photoelectron Spectroscopy (XPS) analysis was performed using an Omicron ESCA system with an EA 125 analyser and XM1000MK II monochromatic X-ray source. Electrochemical measurements were completed using a Gamry Reference 600 potentiostat.

X-ray Photoelectron Spectroscopy (XPS) was employed to probe the chemical nature of the N-rGO material. Fig. 1(a) shows survey scans of a wide spectral region and illustrates the differences in the relative intensities of the characteristic peaks of the elements present. The carbon/oxygen ratio (C/O ratio) for the starting GO is 2.2. After plasma treatment this is increased to 6.3 , signifying the removal of oxygen moieties from the carbon material. The emergence of the N1s peak indicates the presence of nitrogen in the N-rGO. A total nitrogen doping level of $\sim 5 \%$ was achieved. The peaks present at $\sim 450 \mathrm{eV}$ in the GO sample are due to sample mounting and may be ignored. High resolution scans of the $\mathrm{C} 1 \mathrm{~s}$ region for $\mathrm{GO}$ and N-rGO are shown in Fig. 1(b) and (c) with spectral contributions fitted quantifying the nature of the oxygen and nitrogen functional groups on each material. Greater detail and discussion (a)

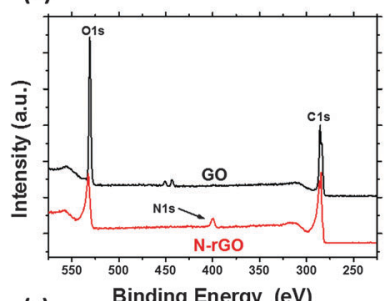

(c) Binding Energy (eV)

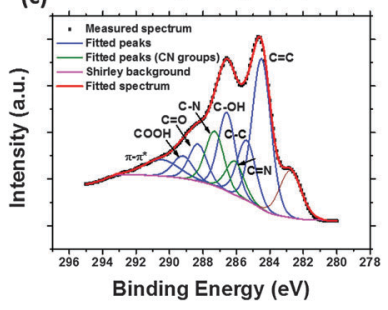

(b)

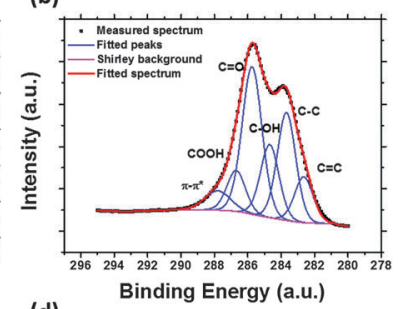

(d)

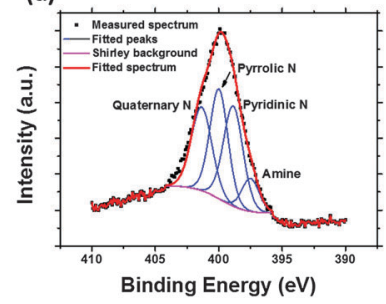

Fig. 1 XPS data for N-rGO. (a) Survey scans normalised to $\mathrm{C} 1 \mathrm{~s}$ for $\mathrm{GO}$ and $\mathrm{N}-\mathrm{rGO}$, (b) GO C1s, (c) N-rGO C1s with fitting, (d) N-rGO N1s. (b)-(d) Display fitted peaks indicating nature of functional groups present.

on the peak assignments is included in the ESI. $\dagger$ After plasma treatment oxygen functional groups are largely removed from the material and nitrogen groups are observed. The N-rGO also displays a greater amount of graphitic carbon than the starting GO, which is heavily $\mathrm{sp}^{3}$-hybridised. Fig. 1(d) shows the N1s peak with spectral contributions from nitrogen in pyridinic, pyrrolic and quaternary configurations with a small contribution from amine functional groups.

Scanning electron microscopy (SEM) was used to characterise the surface of prepared N-rGO electrodes. Representative examples of such films are shown in Fig. 2 for N-rGO spray-coated on ITO/ glass. This shows a uniform film surface free of defects or obvious
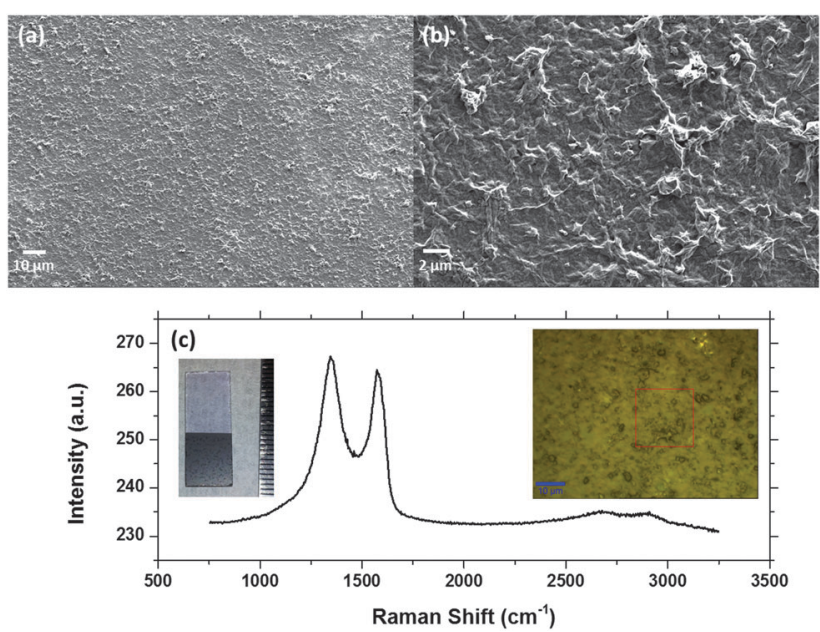

Fig. 2 SEM images of $\mathrm{N}$-rGO spray-deposited on a substrate of ITO/glass at different magnifications, (a) and (b), at an accelerating voltage of $2 \mathrm{kV}$. (c) Average Raman spectrum from 10000 discrete spectra over $20 \times$ $20 \mu \mathrm{m}$ area of deposited film. (Inset, left) photograph of N-rGO spraycoated on ITO/glass substrate as used for electrochemical measurements. (Inset, right) optical micrograph of spray-deposited N-rGO film, the area marked by the red outline indicates the region over which Raman spectra were measured. 
areas of material agglomeration. Some surface roughness due to the flaked nature of the material is inevitable and can be seen in the image, but the long range uniformity of the film is evident.

Raman spectroscopy was utilised to investigate the crystalline nature of the spray-deposited film. Discrete spectra were taken over a $20 \times 20 \mu \mathrm{m}$ region of the film surface and this data was used to generate an average spectrum for the measured area. This is shown in Fig. 2(c). Inset on the left is an optical image of a typical N-rGO electrode. The right inset shows an optical micrograph of the electrode surface with the area over which Raman spectra were measured indicated. The Raman spectrum is typical of a disordered graphitic carbon system with a heavily suppressed 2D band and broad $\mathrm{G}$ and $\mathrm{D}$ band features. ${ }^{34}$ Previous investigations with this material have shown that the Raman spectrum does not change significantly upon plasma treatment. ${ }^{24}$ The spectrum for the spray-deposited film here is largely the same, indicating that no crystalline re-ordering of the material takes place during the electrode fabrication.

Electrochemical characterisation was carried out by cyclic voltammetry (CV) using a three-electrode configuration to investigate any faradaic processes at the electrode surface. Spray deposited electrodes were immersed in $1 \mathrm{M} \mathrm{H}_{2} \mathrm{SO}_{4}$ electrolyte. The N-rGO-coated ITO was then utilised as the working electrode with $\mathrm{Pt}$ and $\mathrm{Ag} / \mathrm{AgCl}$ as the counter and reference electrodes, respectively. Peaks were visible on cyclic voltammograms as shown in Fig. 3(a) and it was found that repeated cycling of the N-rGO electrode caused these peaks to increase in prominence with cycle number. After approximately $600-700$ cycles at a scan rate of $100 \mathrm{mV} \mathrm{s}^{-1}$ the magnitude of the redox peaks was seen to stabilise and remain invariant with cycle number thereafter. The surface-specific capacitance $\left(C_{\mathrm{s}}\right)$ of the electrode was calculated from the coulombic charge stored using formula (1) below.

$$
C_{\mathrm{s}}=\frac{Q}{(E X A)}
$$

The coulombic charge, $Q$, was calculated by integrating the area under the curve for the reverse sweep and dividing by the scan rate. $E$ is the potential window used; $A$ is the area of the working electrode. The electrode area was measured by photographing the electrode adjacent to a known scale and using basic image processing software to calculate the area. The small mass of material deposited on the ITO electrode caused accurate measurement of the mass of N-rGO to be unreliable; resulting in inaccurate determination of capacitance per unit gram. In addition, it has been reported that use of the three electrode configuration can overstate a material's capacitive performance in $\mathrm{F} \mathrm{g}^{-1}$ compared to what is realistically achievable in application configurations. ${ }^{35}$ Hence, in this study, capacitances are dealt with in $\mathrm{F} \mathrm{cm}^{-2}$ when evaluating electrode performances.

The variation of the specific capacitance with cycle number is illustrated in Fig. 3(b). The increase in capacitance up to approximately 700 cycles followed by a levelling off corresponds to the evolution of the redox peaks as shown in Fig. 3(a). Once steady-state performance was achieved, a surface specific
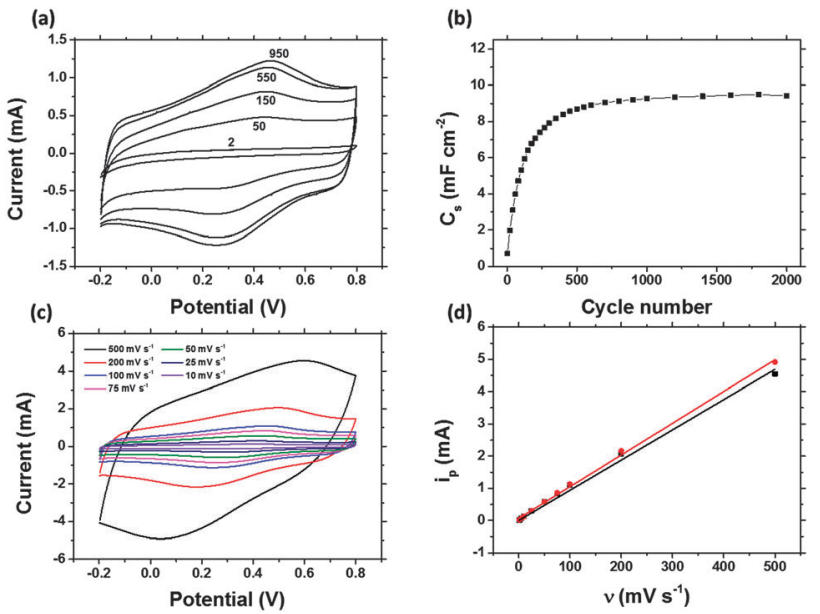

Fig. 3 (a) Voltammograms for repeated cycling of $\mathrm{N}$-rGO working electrode in $1 \mathrm{M} \mathrm{H}_{2} \mathrm{SO}_{4}$ electrolyte at a scan rate of $100 \mathrm{mV} \mathrm{s}^{-1}$. Cycle number corresponding to each voltammogram is included. (b) Surface specific capacitance $\left(C_{s}\right)$ versus cycle number. (c) Voltammetric response of the material at various scans rates. (d) Relationship between peak redox current and scan rate from (c); forward redox peak shown in black, reverse peak shown in red.

capacitance of approximately $9.5 \mathrm{mF} \mathrm{cm}^{-2}$ was observed for the N-rGO. The nature of the redox peaks was further investigated by running cyclic voltammetry at a range of scan rates. First the electrode was cycled at 1000 cycles at $100 \mathrm{mV} \mathrm{s}^{-1}$ to establish the previously observed stable reversible redox behaviour before running cyclic voltammetry at the desired range of scan rates. The resultant voltammograms are displayed in Fig. 3(c), which indicates reversible redox behaviour at lower scan rates. Higher scan rates show less than reversible behaviour, suggesting that not all of these redox events are accessible at these elevated scan rates. This is consistent with previous discussions of faradaic processes at electrode surfaces. ${ }^{36}$ The relationship between the peak redox currents and scan rate for both forward and reverse sweeps is shown in Fig. 3(d). The well-known Randles-Sevcik equation shows that for solution based faradaic processes, the peak redox current will display a linear relationship with the square root of the scan rate. ${ }^{37,38}$ The redox events observed here showed a linear relationship between peak redox current and scan rate. These are attributed to surface faradaic processes, as described in literature, ${ }^{39}$ and are attributed to reversible redox reactions at the functional groups on the N-rGO material. It has previously been reported that retaining some amount of oxygen moieties enhances the capacitive performance of graphene material in addition to the presence of nitrogen groups. ${ }^{40}$ The partially reduced nature of our N-rGO ensures the presence of some residual oxygen functional groups which provide another pseudo-capacitive contribution to the total material capacitance. The capacitance per unit area reported here is approximately twice that reported previously using the same technique for nonfunctionalised liquid phase exfoliated graphene electrodes. ${ }^{33}$ The performance of the N-rGO electrode was further evaluated by comparing it to that of untreated GO. Electrodes fabricated from GO in the same manner as before displayed an appreciably 
inferior performance than the N-rGO in terms of both surface specific capacitance and cycling stability. This is dealt with in detail in the ESI. $\dagger$ Additionally, galvanostatic charge-discharge measurements on N-rGO electrodes further elucidated the charging mechanism of the electrode material and cyclic voltammetry in basic electrolyte was also performed.

\section{Conclusions}

In summary, the capacitive behaviour of N-rGO material, produced by plasma functionalisation of GO, was investigated using a 3-electrode configuration. This allowed faradaic processes at the electrode to be probed in detail. It was determined that surface redox events occur at the functional groups of the $\mathrm{N}$-rGO which contribute a pseudo capacitive mechanism to the charge storage at the electrode. This highlights the importance of the functionalised nature of the N-rGO in enhancing the performance of the material when compared to untreated GO. This redox behaviour took approximately 700 cycles to become fully established before behaving in a fully reversible manner. This is attributed to the electrolyte having to access the total available surface area of the active material and there establish fully reversible redox behaviour.

The ease of electrode fabrication is noteworthy and suggests potential for such materials in supercapacitor applications. Furthermore, it was demonstrated that relatively simple processing can be used to produce large quantities of such N-rGO electrodes; both the plasma treatment and the spray deposition techniques may be up-scaled. This technique negated the requirement for binding agents and conductive additives to be mixed in to the active material. The overall performance of the N-rGO electrodes was shown to be superior to that of electrodes of untreated GO and non-functionalised graphene flakes fabricated by the same means.

\section{Acknowledgements}

This work was supported by the SFI under contracts No. 08/CE/ I1432_S1, PI_10/IN.1/I3030 and the EU under FP7-2010-PPP Green Cars (Electrograph No. 266391). The Advanced Microscopy Laboratory (AML) and its staff are thanked for their assistance in electron microscopy. The authors thank Mr João Coelho for his assistance in sample preparation and Dr Karsten Rode for assistance with XRD measurements.

\section{Notes and references}

1 H.-Y. Kim, K. Lee, N. McEvoy, C. Yim and G. S. Duesberg, Nano Lett., 2013, 13, 2182-2188.

2 G. P. Keeley, A. O'Neill, N. McEvoy, N. Peltekis, J. N. Coleman and G. S. Duesberg, J. Mater. Chem., 2010, 20, 7864 .

3 S. Kumar, N. McEvoy, H. Kim, K. Lee, N. Peltekis, E. Rezvani, H. Nolan, A. Weidlich, R. Daly and G. S. Duesberg, Phys. Status Solidi B, 2011, 248, 2604-2608.
4 Y. Wu, D. B. Farmer, F. Xia and P. Avouris, Proc. IEEE, 2013, 101, 1620-1637.

5 A. L. M. Reddy, A. Srivastava, S. R. Gowda, H. Gullapalli, M. Dubey and P. M. Ajayan, ACS Nano, 2010, 4, 6337-6342. 6 M. Pumera, Chem. Rec., 2009, 9, 211-223.

7 B. P. N. Nguyen, N. A. Kumar, J. Gaubicher, F. Duclairoir, T. Brousse, O. Crosnier, L. Dubois, G. Bidan, D. Guyomard and B. Lestriez, Adv. Energy Mater., 2013, DOI: 10.1002/ aenm.201300330.

8 X. Wang, L. Zhi and K. Mullen, Nano Lett., 2008, 8, 323-327.

9 C. Liu, Z. Yu, D. Neff, A. Zhamu and B. Z. Jang, Nano Lett., 2010, 10, 4863-4868.

10 Y. Shao, J. Sui, G. Yin and Y. Gao, Appl. Catal., B, 2008, 79, 89-99.

11 H. Wang, T. Maiyalagan and X. Wang, ACS Catal., 2012, 2, 781-794.

12 L. Qu, Y. Liu, J.-B. Baek and L. Dai, ACS Nano, 2010, 4, 1321-1326.

13 Y. Wang, Y. Shao, D. W. Matson, J. Li and Y. Lin, ACS Nano, 2010, 4, 1790-1798.

14 H. M. Jeong, J. W. Lee, W. H. Shin, Y. J. Choi, H. J. Shin, J. K. Kang and J. W. Choi, Nano Lett., DOI: 10.1021/nl2009058.

15 K. S. Novoselov, A. K. Geim, S. V. Morozov, D. Jiang, Y. Zhang, S. V. Dubonos, I. V. Grigorieva and A. A. Firsov, Science, 2004, 306, 666-669.

16 X. Li, W. Cai, J. An, S. Kim, J. Nah, D. Yang, R. Piner, A. Velamakanni, I. Jung, E. Tutuc, S. K. Banerjee, L. Colombo and R. S. Ruoff, Science, 2009, 324, 1312-1314.

17 K. V. Emtsev, A. Bostwick, K. Horn, J. Jobst, G. L. Kellogg, L. Ley, J. L. McChesney, T. Ohta, S. A. Reshanov, J. Röhrl, E. Rotenberg, A. K. Schmid, D. Waldmann, H. B. Weber and T. Seyller, Nat. Mater., 2009, 8, 203-207.

18 Y. Hernandez, V. Nicolosi, M. Lotya, F. M. Blighe, Z. Sun, S. De, I. T. McGovern, B. Holland, M. Byrne, Y. K. Gun'Ko, J. J. Boland, P. Niraj, G. Duesberg, S. Krishnamurthy, R. Goodhue, J. Hutchison, V. Scardaci, A. C. Ferrari and J. N. Coleman, Nat. Nanotechnol., 2008, 3, 563-568.

19 M. Lotya, Y. Hernandez, P. J. King, R. J. Smith, V. Nicolosi, L. S. Karlsson, F. M. Blighe, S. De, Z. Wang, I. T. McGovern, G. S. Duesberg and J. N. Coleman, J. Am. Chem. Soc., 2009, 131, 3611-3620.

20 S. Stankovich, D. A. Dikin, R. D. Piner, K. A. Kohlhaas, A. Kleinhammes, Y. Jia, Y. Wu, S. T. Nguyen and R. S. Ruoff, Carbon, 2007, 45, 1558-1565.

21 N. A. Kumar, H.-J. Choi, Y. R. Shin, D. W. Chang, L. Dai and J.-B. Baek, ACS Nano, 2012, 6, 1715-1723.

22 N. A. Kumar, S. Gambarelli, F. Duclairoir, G. Bidan and L. Dubois, J. Mater. Chem. A, 2013, 1, 2789-2794.

23 Y.-C. Lin, C.-Y. Lin and P.-W. Chiu, Appl. Phys. Lett., 2010, 96, 133110.

24 N. McEvoy, H. Nolan, N. Ashok Kumar, T. Hallam and G. S. Duesberg, Carbon, 2013, 54, 283-290.

25 D. Wei, Y. Liu, Y. Wang, H. Zhang, L. Huang and G. Yu, Nano Lett., 2009, 9, 1752-1758. 
26 Z. Jin, J. Yao, C. Kittrell and J. Tour, ACS Nano, 2011, 5, 4112-4117.

27 X. Li, H. Wang, J. T. Robinson, H. Sanchez, G. Diankov and H. Dai, J. Am. Chem. Soc., 2009, 131, 15939-15944.

28 D. Geng, S. Yang, Y. Zhang, J. Yang, J. Liu, R. Li, T.-K. Sham, X. Sun, S. Ye and S. Knights, Appl. Surf. Sci., 2011, 257, 9193-9198.

29 Y. Zhao, C. Hu, Y. Hu, H. Cheng, G. Shi and L. Qu, Angew. Chem., Int. Ed., 2012, 51, 11371-11375.

30 R. I. Jafri, N. Rajalakshmi and S. Ramaprabhu, J. Mater. Chem., 2010, 20, 7114-7117.

31 N. A. Kumar, H. Nolan, N. McEvoy, E. Rezvani, R. L. Doyle, M. E. G. Lyons and G. S. Duesberg, J. Mater. Chem. A, 2013, 1, 4431-4435.

32 W. S. Hummers and R. E. Offeman, J. Am. Chem. Soc., 1958, 80, 1339.
33 B. Mendoza-Sánchez, B. Rasche, V. Nicolosi and P. S. Grant, Carbon, 2013, 52, 337-346.

34 A. C. Ferrari and J. Robertson, Phys. Rev. B: Condens. Matter Mater. Phys., 2000, 61, 14095-14107.

35 M. D. Stoller and R. S. Ruoff, Energy Environ. Sci., 2010, 3, 1294.

36 Z. Lei, L. Lu and X. S. Zhao, Energy Environ. Sci., 2012, 5, 6391-6399.

37 P. Zanello, Inorganic Electrochemistry: Theory, Practice and Applications, Royal Society of Chemistry, 2003.

38 P. T. Kissinger and W. R. Heineman, J. Chem. Educ., 1983, 60, 702.

39 D. Pletcher and S. E. Group, Instrumental methods in electrochemistry, Ellis Horwood, 2001.

40 D. Hulicova-Jurcakova, M. Seredych, G. Q. Lu and T. J. Bandosz, Adv. Funct. Mater., 2009, 19, 438-447. 